\title{
Large Scale Optimization
} IN-G1-CR

(c) whit?

$0.3-3 \gamma$

\section{State of the Art}

Edited by

W. W. Hager

D. W. Hearn

and

P. M. Pardalos

Center for Applied Optimization,

University of Florida, Gainesville, U.S.A.

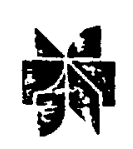

KLUWER ACADEMIC PUBLISHERS DORDRECHT / BOSTON / LONDON 


\title{
A Numerical Comparison of Barrier and Modified Barrier Methods For Large-Scale Bound-Constrained Optimization*
}

\author{
Stephen G. Nash. R. Polyak, and Ariela Sofer \\ George Mason University, Fairfax, VA 22030 USA
}

\begin{abstract}
When a classical barrier method is applied to the solution of a nonlinear programming problem with inequality constraints, the Hessian matrix of the barrier function becomes increasingly ill-conditioned as the solution is approached. As a result, it may be desirable to consider alternative numerical algorithms. We compare the performance of two methods motivated by barrier functions. The first is a stabilized form of the classical barrier method, where a numerically stable approximation to the Newton direction is used when the barrier parameter is small. The second is a modified barrier method where a barrier function is applied to a shifted form of the problem, and the resulting barrier terms are scaled by estimates of the optimal Lagrange multipliers. The condition number of the Hessian matrix of the resulting modified barrier function remains bounded as the solution to the constrained optimization problem is approached. Both of these techniques can be used in the context of a truncatedNewton method, and hence can be applied to large problems, as well as on parallel computers. In this paper, both techniques are applied to problems with bound constraints and we compare their practical behavior.
\end{abstract}

Keywords: nonlinear programming, barrier method, modified barrier method, Newton's method, truncated-Newton method, large-scale optimization.

- The authors were partially supported by National Science Foundation grant DDM-9104670. R. Polyak was partially supported by NASA contract NAG3-1397 and National Science Foundation grant DMS-9300962.

IV. IV. Hager et al. (eds.). Large Scaie Optimization: State of the An, 319-338.

1994 Kluwer Academic Publishers. Printed in the Netheriands. 


\section{Introduction}

We will examine the solution of nonlinear programming problems of the form

$$
\begin{array}{ll}
\operatorname{minimize} & f(x) \\
\text { subject to } & c_{i}(x) \geq 0, \quad i=1, \ldots, m .
\end{array}
$$

Here $x=\left(x_{1}, \ldots, x_{n}\right)^{r}$ and the functions $f$ and $\left\{c_{i}\right\}$ will be assumed to be twice continuously differentiable. We have in mind cases where $n$ is large.

The methods we will consider for solving (1) will be based on classical barrier functions. The constrained problem is converted to a sequence of unconstrained problems. If the logarithmic barrier function is used. then the unconstrained problems
have the form

$$
\beta(x, \mu)=f(x)-\mu \sum_{i=1}^{m} \ln \left(c_{1}(x)\right),
$$

involving a "barrier parameter" $\mu>0$. If $x^{*}(\mu)$ denotes a minimizer of $\beta(x, \mu)$ then, under appropriate conditions, it can be shown that (as $\mu \rightarrow 0$ ) any limit point $x^{*}$ of the sequence $\left\{x^{*}(\mu)\right\}$ is a solution of (1) (see Fiacco and McCormick[5]). In addition, the associated Lagrange multiplier estimates converge to the Lagrange multipliers at
$x^{*}$.

It is well known that the Hessian matrix of the barrier function becomes increasingly ill-conditioned as $\mu \rightarrow 0$ and a solution to (1) is approached. (This will be discussed in more detail in Section 3.) More specifically, if $k$ constraints are binding

$$
\lim _{\mu \rightarrow 0^{+}} \operatorname{cond}\left(\nabla^{2} \beta\left(x^{*}(\mu), \mu\right)\right)=+\infty .
$$

Thus the classical barrier method "breaks down" as the method converges to the solution of the original constrained problem.

We will examine two approaches that avoid this "structural" ill-conditioning (i.e., the ill-conditioning associated with the method, as distinct from the conditioning of the underlying optimization problem). Both approaches solve a sequence of unconstrained optimization problems involving a (possibly modified) barrier function. The first uses a numerically stable approximation to the Newton direction for the classical barrier function (Nash and Sofer [14]). The second uses Polyak's modified barrier method [17], which incorporates an explicit representation of the Lagrange multipliers with an extension of the feasible region. Combined, these features can alleviate the problem of ill-conditioning, and improve the overall rate of convergence.

In this paper, each of these unconstrained problems will be solved using a truncated-Newton method. In this method, the Newton equations for a search direction are solved approximately using the conjugate-gradient method. Why choose a truncatedNewton method? It is a Newton-type method, that requires only first derivatives (although second derivatives may be utilized if desired); it has low storage costs; 
$\geq$ form

1 to be twice

ssical barrier inconstrained ned problems

$\beta(x, \mu)$ then, it point $x^{*}$ of 1. In addition, multipliers at

omes increas(This will be ts are binding

iverges to the

ditioning (i.e., onditioning of nce of unconfunction. The or the classical odified barrier ange multipli; can alleviate gence.

ssing a trunca$\mathrm{h}$ direction are ie a truncatedrst derivatives storage costs;

it can be adapted to solve nonconvex problems; and it vectorizes well. Thus the method reduces the costs of Newton's method while maintaining rapid convergence, and is therefore suitable for large-scale problems. In practice the method has proven to be robust, effective and competitive on a wide set of unconstrained minimization problems.

The stabilized barrier method is the sarne as in Nash and Sofer [14], although it is tested here on a larger set of problems (and using a different computer). The modified barrier method software is new, although it was obtained by modifying the software for the stabilized barrier method. Because much of the software for the two methods is the same, we believe that this gives a clearer comparison of the properties of the two methods.

We will compare the performance of the two methods on a set of 1000-variable problems with bound constraints. Preliminary computational experience with modified barrier methods, using either a BFGS quasi-Newton method or a conjugate gradient algorithm as the unconstrained minimization technique, is presented by Breitfeld and Shanno [3]. Their paper presents numerical results for a set of problems that are small, but have true nonlinear constraints.

\section{The Truncated-Newton Method}

In both the modified barrier method and the stabilized barrier method, the unconstrained subproblems will be solved using a modifed version of the truncated-Newton software described in Nash and Nocedal [11]. A summary of this method will be given here, as applied to an unconstrained problem

$$
\underset{x}{\operatorname{minimize}} f(x) \text {. }
$$

The notation $\nabla f=\nabla f(x)$ is used for the gradient of $f$ evaluated at a point $x$.

Given some initial guess $x_{0}$, at the $j$-th iteration the new estimate $\hat{x}$ of the solution is given by

$$
\hat{x}=x+\alpha p .
$$

The search direction $p$ must satisfy $p^{T} \nabla f<0$ (i.e., it is a descent direction for $f$ at the point $x$ ).

The step length $\alpha>0$ is chosen to guarantee that $f(\hat{x})<f(x)$, along with other conditions designed to guarantee convergence to a local minimizer (see Ortega and Reinboldt [16]). The particular line search algorithms used are discussed below.

The search direction $p$ is computed as an approximate solution of the Newton equations

$$
\left(\nabla^{2} f\right) p=-\nabla f
$$

where $\nabla^{2} f \equiv \nabla^{2} f(x)$ is the Hessian matrix of second derivatives at the current point $x$. The approximate solution is obtained by applying the conjugate-gradient method 
to (2). This iterative method is "truncated" before the exact solution is obtained. On parallel computers, a block conjugate gradient method could be used to solve (2), resulting in a parallel barrier method (see [13]). This idea has been applied to bound-constrained problems for the stabilized barrier method in (10).

The conjugate-gradient method corresponds to minimizing the quadratic model $Q(p)=\frac{1}{2} p^{T} \nabla^{2} f p+p^{T} \nabla f$ as a function of $p$ over a sequence of subspaces of increasing dimension. These are called the Krylov subspaces.

The truncated-Newton software used here includes automatic preconditioning strategies designed to accelerate convergence of the conjugate-gradient method. These were not modified in the computational tests used in this paper, because of the special form of the bound constraints. For problems with more general constraints, it is likely that the preconditioners would have to be adjusted to take into account the special structure of the barrier subproblems. Techniques for doing this are discussed by Nash and Sofer in [15].

\section{The Stabilized Barrier Method}

The discussion here is adapted from [14], and presents a summary of the stabilized barrier method. For a more complete discussion, the reference should be consulted.

We will assume that a strictly feasible initial guess of the solution has been provided. For problems with bound constraints, such a point can be easily found. In addition, we make the following standard assumptions: (a) the feasible set is compact and has a non-empty interior; (b) a solution $x^{*}$ lies in the closure of the interior of the feasible region; (c) $x^{*}$ is a regular point of the constraints (i.e., the gradients of the active constraints at $x^{*}$ are linearly independent) which satisfies the second-order sufficiency conditions for optimality (see Fiacco and McCormick [5]).

The logarithmic barrier method converts the problem (1) to a sequence of unconstrained problems:

$$
\underset{x}{\operatorname{minimize}} \beta(x, \mu)=f(x)-\mu \sum_{i=1}^{m} \ln \left(c_{i}(x)\right)
$$

for a sequence of positive barrier parameters $\mu \rightarrow 0$. Let $x^{*}(\mu)$ denote an unconstrained minimizer of $\beta(x, \mu)$. Under quite mild conditions it can be shown that any limit point $x^{*}$ of the sequence $x^{*}(\mu)$ is a solution of (1). Furthermore if we define

$$
\lambda_{i}(\mu) \equiv \mu / c_{1}\left(x^{*}(\mu)\right),
$$

then as $x^{*}(\mu) \rightarrow x^{*}, \lambda(\mu) \rightarrow \lambda^{*}$, where $\lambda^{*}$ is the vector of Lagrange multipliers corresponding to $x^{*}$ (see [5]).

The Newton direction for the barrier subproblem (3) at the point $x$ is obtained by solving

$$
B p=-b
$$


ion is obtained. re used to solve been applied to zuadratic model ces of increasing

preconditioning imethod. These ause of the speil constraints, it into account the is are discussed

of the stabilized $\mathrm{d}$ be consulted. in has been proeasily found. In le set is compact ff the interior of the gradients of the second-order uence of uncon-

note an unconshown that any e if we define

ange multipliers nt $x$ is obtained where $b$ and $B$ are the gradient and Hessian matrix respectively, of the logarithmic barrier function:

$$
\begin{aligned}
& b=\nabla f-\mu \sum_{i=1}^{m} \frac{\nabla c_{i}}{c_{i}}, \\
& B=\nabla^{2} f-\mu \sum_{i=1}^{m} \frac{\nabla^{2} c_{i}}{c_{i}}+\mu \sum_{i=1}^{m} \frac{\nabla c_{i} \nabla c_{i}^{T}}{c_{i}^{2}} .
\end{aligned}
$$

(To simplify the formulas, $f$ is written for $f(x)$, etc.) If we define $\lambda_{i}=\mu / c_{i}$, then $(t)$ can be expressed in the form

$$
\begin{aligned}
& b=\nabla f-\sum_{i=1}^{m} \lambda_{i} \nabla c_{i}, \\
& B=\nabla^{2} f-\sum_{i=1}^{m} \lambda_{i} \nabla^{2} c_{i}+\frac{1}{\mu} \sum_{i=1}^{m} \lambda_{i}^{2} \nabla c_{i} \nabla c_{i}^{T} .
\end{aligned}
$$

The final term in (5) reveals the ill-conditioning in the barrier subproblem. If a constraint is active at the solution, and its corresponding Lagrange multiplier is non-zero. then the ratio $\lambda_{1}^{2} / \mu \rightarrow \infty$ as $\mu \rightarrow 0$. Thus the Hessian matrix becomes progressively more ill conditioned as the solution is approached. This ill-conditioning was noted by Murray in [\$].

The stabilized barrier method avoids this ill conditioning by using an approximation to the Newton direction for the barrier function. This approximation differs from the Newton direction by terms of $O(\mu)$ and so becomes more accurate as $\mu \rightarrow 0$. The approximation is obtained by examining the range- and null-space components of the search direction. defined in terms of a "working set" of constraints, analogous to the working set used in an active-set method for constrained optimization (see, for example. [6]). The approach we propose does not require that the Hessian matrix of the barrier be formed explicitly. A different approach that avoids the ill conditioning but that requires explicit matrix factorizations is described by Wright in [18].

To develop the formulas for the search direction, we define $I$ to be the index set of those constraints that contribute to the ill conditioning of the Hessian matrix. This set is a prediction of the set of constraints that are binding at the solution of (1). Let $V$ be the matrix whose columns are the gradients of the constraints in $\mathcal{I}$. and assume that $N$ has full rank. We define $D=\operatorname{diag}\left(\lambda_{i}^{2}, i \in I\right)$, and choose $Z$ as a basis for the null space of $N^{T}$. Let $V^{\#}$ be a pseudo-inverse for $V$. (For bound-constrained problems. the columns of.$V$ and $Z$ are just columns of the identity matrix.) Finally, define

$$
H=\nabla^{2} f-\sum_{i=1}^{m} \lambda_{i} \nabla^{2} c_{i}+\frac{1}{\mu} \sum_{i \in T} \lambda_{i}^{2} \nabla c_{i} \nabla c_{i}^{T},
$$

i.e., the "good" part of the Hessian matrix $B$, omitting the ill-conditioned terms. Using these definitions the Newton direction can be approximated via

$$
p \approx p_{1}+\mu p_{2},
$$


where

$$
\begin{aligned}
p_{1} & =-Z\left(Z^{T} H Z\right)^{-1} Z^{T} b, \\
\hat{\lambda} & =N^{\#}\left(H p_{1}+b\right), \\
p_{2} & =-\left(N^{\#}\right)^{T} D^{-1} \hat{\lambda} .
\end{aligned}
$$

These formulas correspond to an $O(\mu)$ approximation to the Newton direction. (A related stabilized formula for the search direction was derived by Murray in (8].)

The formulas (6) only require $\left(Z^{T} H Z\right)^{-1}$. In our algorithm this is implemented by applying the conjugate-gradient method to

$$
Z\left(Z^{T} H Z\right)^{-1} Z^{T} p_{1}=-b
$$

with the iteration truncated as in the unconstrained case. The costs of finding the search direction in this approach are comparable to those of a naive barrier method that does not deal with the ill conditioning. The approximate direction obtained using the formulas (6), together with a truncated conjugated-gradient iteration, can be shown to be a descent direction for the barrier function under appropriate assumptions.

A number of computational enhancements were used to improve the performance of the stabilized barrier method. These are discussed briefly in Section 5 .'

\section{The Modified Barrier Method}

We now describe the modified barrier method for the constrained problem (1). An extensive discussion of the theory of modified barrier methods can be found in the paper by Polyak [17].

At each major iteration of the modified barrier method the unconstrained problem

$$
\underset{x}{\operatorname{minimize}} \mathcal{M}(x, \lambda, \mu)
$$

is solved where

$$
\mathcal{M}(x, \lambda, \mu)=f(x)-\mu \sum_{i=1}^{m} \lambda_{i} \psi\left(\mu^{-1} c_{i}(x)+1\right),
$$

and the solution $\hat{x}$ is used to update $\left\{\lambda_{i}\right\}_{i=1}^{m}$ via

$$
\dot{\lambda}_{i}=\lambda_{i} \psi^{\prime}\left(\mu^{-1} c_{i}(\hat{x})+1\right) \text {. }
$$

The parameters $\left\{\lambda_{i}\right\}$ are estimates of the Lagrange multipliers at the solution $x^{*}$. The function $\psi$ is a monotone, strictly concave, and twice continuously differentiable function defined on the interval $(0,+\infty)$; one possible choice is $\psi(\cdot)=\ln (\cdot)$, although 
rak, and A. Sofer

ton direction. (A Iurray in [8].) is is implemented

osts of finding the ve barrier method ction obtained ust iteration, can be propriate assump-

re the performance ction 5 .

1 problem (1). An in be found in the ,nstrained problem

at the solution $x^{*}$. cously differentiable $\cdot)=\ln (\cdot)$, although ur algorithm will use a more complicated definition of $\psi$. It is also possible to use the inverse function $\psi(\cdot)=1 /(\cdot)$ although this choice is not tested here.

If, for example, $\psi(\cdot)=\ln (\cdot)$, then the feasible region for (1) is equivalent to the set

$$
\left\{x: \mu \psi\left(\mu^{-1} c_{t}(x)+1\right) \geq 0\right\} \text {. }
$$

Thus the modified barrier function is the classical Lagrangian for the problem (1) with the constraints expressed in this equivalent form. The use of the barrier term

$$
\psi\left(\mu^{-1} c_{i}(x)+1\right)
$$

corresponds to perturbing the constraints so that they have the form

$$
c_{i}(x) \geq-\mu
$$

This represents an expansion of the feasible region. Hence the implied "feasible region" for the modified barrier subproblem varies with the barrier parameter $\mu$.

Unlike the classical logarithmic barrier function, the modified barrier function and its derivatives exist at a solution $x^{*}$ for any positive barrier parameter $\mu$. In particular, if $\lambda^{*}$ is the vector of Lagrange multipliers corresponding to $x^{*}$, and if $\psi(\cdot)=\ln (\cdot)$, then the modified barrier function has the following properties for any $\mu>0$ :

$$
\begin{array}{ll}
\text { P1. } & \mathcal{M}\left(x^{*}, \lambda^{*}, \mu\right)=f\left(x^{*}\right) \\
\text { P2. } & \nabla_{x} \mathcal{M}\left(x^{*}, \lambda^{*}, \mu\right)=\nabla f\left(x^{*}\right)-\sum_{i=1}^{m} \lambda^{*} \nabla c_{i}\left(x^{*}\right)=0 \\
\text { P3. } & \nabla_{x x^{2}}^{2} \mathcal{H}\left(x^{*}, \lambda^{*}, \mu\right)=\nabla^{2} f\left(x^{*}\right)-\sum_{i=1}^{m} \lambda_{i}^{*} \nabla^{2} c_{i}\left(x^{*}\right)+\mu^{-1} \sum_{i=1}^{m} \lambda_{i}^{*} \nabla c_{i}\left(x^{*}\right) \nabla c_{i}\left(x^{*}\right)^{T}
\end{array}
$$

When the problem is a convex program, it follows from P2 that

$$
\text { P4. } \quad x^{*}=\arg \min \left\{\mathcal{M}\left(x, \lambda^{*}, \mu\right)\right\} \quad \text { for any } \mu>0 \text {. }
$$

This means that if the optimal Lagrange multipliers $\lambda^{*}$ are known, one can solve the constrained problem (1) using a single unconstrained optimization problem regardless of the value of the barrier parameter. Moreover, if the constrained optimization problem is nonconvex but the second-order sufficiency and strict complementarity conditions are satisfied at $x^{*}$ then there exists a $\vec{\mu}$ and a $\bar{\rho}>0$ such that:

$$
\text { P5. } \quad \min \operatorname{eig} \nabla_{x x}^{2} \mathcal{M}\left(x^{*}, \lambda^{*}, \mu\right) \geq \bar{\rho} \quad \text { for } \mu<\vec{\mu} \text {. }
$$

Thus it is again possible to solve (1) using a single unconstrained optimization problem of the form (7) provided that the barrier parameter is sufficiently small. Of course, in practice only a local minimizer may be found. 
Polyak [17] has shown that if the initial Lagrange multipliers are positive, and the barrier parameters are below some threshhold value $\bar{\mu}$, then the method converges. Furthermore, for sufficiently small $\mu$, the successive iterates satisfy

$$
\max \left\{\left\|\hat{x}-x^{*}\right\| \cdot\left\|\hat{\lambda}-\lambda^{*}\right\|\right\} \leq c \mu\left\|\lambda-\lambda^{*}\right\| .
$$

The constant $c>0$ is independent of $\mu \leq \bar{\mu}$.

For a convex programming problem it is possible to prove a further result. Under mild conditions on the primal and dual feasible regions the modified barrier method converges for any fixed positive value of the barrier parameter $\mu$, provided that the initial vector of Lagrange multipliers is positive (see Jensen and Polyak [7]). This is indeed a strong result. Unlike the classical barrier method, where convergence is obtained by driving the barrier parameter to zero, in the modified barrier method convergence will occur regardless of the value of the barrier parameter

The result (9) shows that the modified barrier method converges at a superlinear rate if the barrier parameter is changed from subproblem to subproblem in such a way that $\mu \rightarrow 0$. However it is not necessary that $\mu \rightarrow 0$ in order to achieve convergence; it is only necessary that $\mu$ be reduced below the threshhold value $\bar{\mu}$. Thus the condition number of the Hessian matrix of the modified barrier function can remain bounded as the solution is approached, unlike in the classical case.

On practical problems, it is not possible to know a priori whether the initial parameter chosen is indeed below the threshhold $\bar{\mu}$, and therefore a general-purpose code for solving (1) must also include some mechanism for reducing the barrier parameter. However some caution is required. If a solution $\hat{x}(\mu)$ to a modified barrier subproblem has been found, and $\mu$ is reduced to a new value $\hat{\mu}$ it is possible that $\hat{x}(\mu)$ will be "infeasible" for the new subproblem:

$$
c_{i}(\hat{x}(\mu)) \geq-\hat{\mu} \text {. }
$$

Suppose that the function $\psi$ is chosen as $\dot{\psi}(\cdot)=\ln (\cdot)$. Then if $\dot{\mu}<\mu$ and $c_{i}(\hat{x})<0$ it is possible that

$$
\psi\left(\hat{\mu}^{-1} c_{i}(\hat{x})+1\right)=\ln \left(\hat{\mu}^{-1} c_{i}(\hat{x})+1\right)
$$

might be undefined. This limits the flexibility of the modified barrier method (it limits how quickly $\mu$ can be reduced) and it can greatly complicate software for this algorithm, particularly if the constraints are nonlinear (see also [3!).

For this reason we have chosen to use a more elaborate definition of the function $\psi$, a definition that varies with the value of $\mu$. In our implementation we use a modification that has been suggested by Ben-Tal, Tsibulevskii and Yusefovich [2]. Let $t=c_{i}(x)$. If $t \geq-\mu / 2$ then we define

$$
\psi\left(\mu^{-1} t+1\right)=\ln \left(\mu^{-1} t+1\right) .
$$

If $t<-\mu / 2$ then we define

$$
\psi\left(\mu^{-1} t+1\right)=q(t)
$$


positive, and the rethod converges.

her result. Under d barrier method rovided that the ?olyak (7]). This re convergence is d barrier method ter

$s$ at a superlinear lem in such a way ve convergence; it hus the condition remain bounded

ler the initial pa- general-purpose g the barrier pamodified barrier rossible that $\hat{x}(\mu)$

เ and $c_{\mathrm{r}}(\hat{x})<0$ it

rrier method (it software for this

1 of the function tation we use a 1 Yusefovich [2]. where $q(t)$ is a quadratic function for which $q(-\mu / 2), q^{\prime}(-\mu / 2)$, and $q^{\prime \prime}(-\mu / 2)$ match the corresponding values for the logarithm function at the point $t=-\mu / 2$. Since the quadratic function does not have a singularity at $-\mu$ (or at any other point), the barrier parameter can be reduced at any desired rate without worrying whether the modified barrier function will become undefined or singular.

Our software for the modified barrier algorithm was obtained by adapting the software for the stabilized barrier method. The underlying unconstrained optimization method is the same truncated-Newton method. More specific details (chosen as a result of considerable numerical testing) are discussed in Section 5.

\section{Implementation}

A number of computational enhancements were used to improve the performance of the stabilized barrier method. We give a brief description of these enhancements and discuss their effect when implemented within a modified barrier method.

\subsection{The Line Search}

Because the logarithmic barrier function has a singularity at the boundary of the feasible region, standard line search algorithms based on low-order polynomial interpolation may not be effective. For example, in implementing an inverse cubic interpolation line search we found that an unusually large proportion (often more than $50 \%$ ) of the overall computational effort was spent within the line search. Replacing this line search by an Armijo-type strategy reduced the fraction of time spent in the line search but increased the overall computational effort substantially.

For this reason we implemented a special line search devised by Murray and Wright [9] specifically for the logarithmic barrier function. This line search approximates the barrier function along the search direction with a one-dimensional function consisting of a quadratic term plus a logarithmic singularity. We have found this line search to he effective when implemented within a classical barrier method. For example, on a set of problems tested in [14], the special line search led to a $27 \%$ reduction in the overall computational effort.

The special line search was not as beneficial when implemented within a modified barrier method. This may be due to the fact that our elaborate definition of $\psi$ no longer has a logarithmic singularity. The line search currently implemented in our software is a standard line search for unconstrained minimization based on inverse cubic interpolation with an acceptance test based on a Wolfe condition (the "default" line search for the truncated-Newton method). 


\subsection{Extrapolation}

A (classical) barrier method can be improved significantly by extrapolation. This techriique uses the solutions of the subproblems for previous barrier parameters to fit a low-order polynomial to the barrier trajectory. The polynomial is then used to a better initial guess for the subproblem for the new barrier parameter. This provides

Our ow axperience indicates that subst

quadratic extrapolation, and that substantial gains may be obtained by using cubic extrapolation instead. The modest additional gains may be obtained by using

Our attempts to accelerate the or cubic extrapolation were not successful barrier using either linear, quadratic modified barrier subproblems do not lie on a The reason is that the solutions to the is true for the classical barrier function. used to obtain the initial guess for a new Thus in the current code, no extrapolation is subproblem is used as an initial guess without modification.

\subsection{Initializing the Barrier Parameter}

The selection of the initial barrier parameter can have a dramatic effect on the running time of the algorithm. A parameter that is too small may cause the subproblem to require the solution of too many subsequent subproblems.

The best initialization scheme that

a heuristic that attempts to find that we found for the stabilized barrier method is on the barrier trajectory which is "closest" parameter corresponding to the point does not appear to be effective for the modified barrier method: The same scheme parameter tends to be "too large." Better results were obtained the resulting initial barrier parameter to a relatively small value.

\subsection{Preconditioning}

To be effective, a truncated-Newton method must use preconditioning. The software for the truncated-Newton method uses a preconditioner based on a limited-memory in turn is scaled by a diagonal from consecutive truncated-Newton iterations, which conjugate gradient iterations. The stabilized the Hessian matrix obtained from the ditioner from one subproblem as the initial barrier software uses the final preconThe modified barrier method uses the same strategy. 
ipolation. This r parameters to is then used to $\therefore$ This provides

tained by using , tained by using c extrapolation. near, quadratic solutions to the zterized by $\mu$, as extrapolation is t to the previous

ton the running : subproblem to is too large may

arrier method is ng to the point te same scheme resulting initial sting the initial

g. The software limited-memory terations, which stained from the he final preconext subproblem.

\section{A Vumerical Comparison of Barrier and Modified Barrier Methods}

\subsection{Customized Matrix-Vector Product}

The stabilized barrier method uses a customized matrix-vector product for the conjugate-gradient iteration that isolates the terms associated with the working set $I$. This is necessary so that rounding errors from the ill-conditioned terms do not contaminate the well-conditioned terms in the Hessian matrix, and hence destroy the effects of the stabilized approximation to the Vewton direction.

If $B$ denotes the Hessian matrix of the barrier function then the product $B u$ is computed via the formula

$$
B u=\left(\nabla^{2} f\right) u-\mu \sum_{i=1}^{m} \frac{\left(\nabla^{2} c_{i}\right) u}{c_{i}}+\mu \sum_{i=1}^{m} \frac{\left(\nabla c_{i}^{T} u\right) \nabla c_{i}^{T}}{c_{i}^{2}} .
$$

The terms $\left(\nabla^{2} f\right) u$ and $\left(\nabla^{2} c_{i}\right) u$ are computed via finite differencing:

$$
\left(\nabla^{2} f\right) u \approx \frac{\nabla f(x+h u)-\nabla f(x)}{h}
$$

where $h$ is (approximately) the square root of the machine precision. It is not safe to apply finite differencing directly to $B u$ because of the singularity of the logarithmic function. The final summation in the formula for $B u$ is computed straightforwardly from the formulas above. When the stabilized formulas for the search direction are used, the product $H u$ must be computed. This is done in the same way, except that the ill-conditioned terms are omitted from the final summation.

The modified barrier uses a similar approach, except applied to the Hessian of the modified barrier function.

\section{Computational Tests}

In this section we compare the modified barrier method and the stabilized barrier method on a set of test problems with bound constraints.

Many of our test problems are derived from a set of unconstrained optimization problems; see Table 1. For more detailed information about problems 1-52, see [11]. Problems 54 and 55 are from [4]. The final two problems are from release 2 of the Minpack-2 collection [1]. They are DPJBFG (pressure in a journal bearing) and DEPTFG (elastic-plastic torsion). These are the only two minimization problems in this collection which have bound constraints that are binding at the solution. For problem DPJBFG we set $n x=n y=\sqrt{n}$, ecc $=0.1$, and $b=10$. For problem DEPTFG we set $n x=n y=\sqrt{n}$, and $c=5$.

The constrained problems $1-55$ are as in [14]. In each case, we first solve the corresponding unconstrained problem, computing $\hat{x}$ satisfying

$$
\|g(\hat{x})\|_{\infty} \leq 10^{-5}(1+|f(\dot{x})|)
$$




\begin{tabular}{|c|c|c|}
\hline Problem & Name & $n$ \\
\hline 1 & Calculus of variations 1 & 100,1000 \\
\hline 2 & Calculus of variations 2 & 100,1000 \\
\hline 3 & Calculus of variations 3 & 100.1000 \\
\hline 6 & Generalized Rosenbrock & 100,1000 \\
\hline 8 & Penalty 1 & 1000 \\
\hline 9 & Penáty 2 & 100 \\
\hline 10 & Penalty 3 & 1000 \\
\hline 12 & Quadratic & 1000 \\
\hline 28 & Extended Powell singular & 1000 \\
\hline 30 & Trigonometric & 100 \\
\hline 31 & Brown almost-linear & 100 \\
\hline 38 & Tridiagonal 1 & 1000 \\
\hline 39 & Linear minimal surface & 961 \\
\hline 40 & Boundary-value problem & 1000 \\
\hline 41 & Broyden tridiagonal nonlinear & 1000 \\
\hline 42 & Extended ENGVL1 & 1000 \\
\hline 43 & Ext. Freudenstein and Roth & 1000 \\
\hline 45 & Wrong extended Wood & 1000 \\
\hline 48 & Extended Rosenbrock & 1000 \\
\hline 49 & Extended Powell & 1000 \\
\hline 50 & Tridiagonal 2 & 1000 \\
\hline 51 & Trigonometric & 1000 \\
\hline 52 & Penalty 1 (2nd version) & 1000 \\
\hline 54 & Toint 61 & 1000 \\
\hline 55 & Toint 62 & 1000 \\
\hline 102 & Minpack-2 (DJOURB) & 100 \\
\hline 105 & Minpack-2 (DTOR) & 100. 1024 \\
\hline
\end{tabular}

Table 1: List of test problems. 
sing the standard initial point $x_{0}$. Lower and upper bounds are then derived from If $i$ is odd then

$$
-100 \leq x_{i} \leq 100
$$

$i f$ is a multiple of $t$ then

$$
(\hat{x})_{i}+0.1 \leq x_{i} \leq(\hat{x})_{i}+10.0
$$

if $i$ is even but not a multiple of 4 then

$$
(\hat{x})_{i}-10.0 \leq x_{i} \leq(\hat{x})_{i}-0.1
$$

Then a strictly feasibly initial point for the barrier method is generated. If $\left(x_{0}\right)_{1}<\ell_{\mathrm{s}}$ then $\left(x_{0}\right)_{i}=\ell_{i}+0.5 ;$ if $\left(x_{0}\right)_{i}>u_{i}$ then $\left(x_{0}\right)_{i}=u_{i}-0.5$. If $\left(x_{0}\right)_{i}=\ell_{i}$ then $\left(x_{0}\right)_{i}=$ $i_{i}+10^{-4} ;$ if $\left(x_{0}\right)_{i}=u_{i}$ then $\left(x_{0}\right)_{i}=u_{i}-10^{-4}$. Then $x_{0}$ is used as the initial point for the barrier method.

The algorithms were programmed in Fortran 77 and the runs were made using luuble precision on an IBM $320 \mathrm{H}$ RISC workstation. The "stabilized" algorithm uses the stabilized formula for the Newton direction when $\mu$ is small; the "modinei" algorithm uses the modified barrier method. The two methods incorporate the inhancements described in Section 5.

Both methods compute a search direction using a conjugate-gradient iteration terminated as in [12], using a rule based on the value of the quadratic model with tolerance 0.5 . Both barrier methods were terminated when the norm of the complementary slackness vector (scaled by $1+|f(x)|$ ) was less than $\epsilon_{1}=10^{-8}$, and when the norm of the Lagrangian gradient (also scaled by $1+|f(x)|$ ) was less than $\epsilon_{2}=10^{-5}$. I $n$ addition, we required that the solution from the modified barrier method not be infeasible with respect to any constraint by more than $\epsilon_{1}=10^{-8}$.

We list here some details of the implementation for the stabilized barrier method. For further information, see Nash and Sofer [14].

- The line search was terminated using an Armijo-type test with parameter $\eta=0.2$.

- The barrier parameter was updated using $\mu_{k+1}=\mu_{k} / 10$.

- The truncated-Newton method (for a given $\mu$ ) was terminated when the norm of the gradient (scaled as above) was less than $\epsilon_{3}=10^{-3}$, and when the smallest Lagrange multiplier estimate was greater than $-\epsilon_{4}$, where $\epsilon_{4}=10^{-6}$.

- The stabilized formula for the Newton direction was invoked when the norm of the scaled complementary slackness vector was less than $\epsilon_{5}=10^{-4}$.

We made many test runs using the modified barrier method, and some of the more interesting ones are described below. However, we will only be providing detailed rcsults for the best of these runs, for which the following parameter settings were used:

- The line search was terminated using a Wolfe-type test with parameter $\eta=$ 0.25 . 

- the initial barrier parameter was the same for all test problems, $\mu_{0}=10^{-3}$;
the barrier parameter was updated using $\mu_{k+1}=\mu_{k} / 2$;

- the initial Lagrange multiplier estimates were chosen to be $\lambda_{i}=1, i=1, \ldots, m_{\text {; }}$

- for the first subproblem, the truncated-Newton method was terminated when the norm of the scaled gradient was less than $\epsilon_{3}=10^{-3}$;

- for subsequent subproblems, the truncated when the norm of the scaled thrancated-Newton method was terminated For a particular algorithm, scaled gradient was less than $\epsilon_{3}=10^{-6}$; of the test problems. The algorithms set of parameter settings was used to solve all

The detailed results are given in wable not "tuned" to particular problems. the barrier method, but not the costs a and the bounds (that is, the costs of solving with determining the initial point ignored). An entry in the table consists of four numitial unconstrained problem are outer iterations), "ls" (the number of gradient evambers: "it" (the total number of "cg" (the number of gradient evaluations used evaluations used in the line search), Hessian-vector products), and "total" (the sum of "s inner iteration to compute the

The results in Table 2 indicate that the sum of " $(\mathrm{s}$ " and " $\mathrm{cg}$ "). better than the stabilized barrier method modified barrier method performs notably method requires only $74 \%$ as many trun on these problems. The modified barrier many gradient evaluations. In truncated-Newton iterations, and only $68 \%$ as stabilized barrier method only examining individual problems it is seen that the problems: problems $1(n=100,1000)$, modified barrier method on 9 of the 33 $(n=100)$. We should $(n=100,1000), 12,42,49,54,102(n=100,1024), 105$ our desire to use a single set of parameter sindividual results are a by-product of parameter settings that minimize the grand tottings for all test problems. By seeking the method on individual problems can deteral for the entire test set, the behavior of parameters for these problems it is possible torate. In particular, by fine-tuning the the modified barrier method (at the cost of obtain much better performance from

For the other computational tests of the poorer performance on other problems). the totals for the four table entries. Note that for thed barrier method we will only list we were able to find, the totals were

\section{$\left(\begin{array}{llll}1592 & 3361 & 7613 & 10974\end{array}\right)$}

We experimented with solving the first subproblem both more and less accurately, but this was less effective. When the initial subproblem was terminated when the norm of the scaled gradient was less than $\epsilon_{3}=10^{-2}$ (instead of $\epsilon_{3}=10^{-3}$ ) then the
totals were:

\section{$\left(\begin{array}{llll}1748 & 3463 & 8705 & 12168\end{array}\right)$}

Similar results were obtained when the first subproblem was terminated after a fixed number (6) truncated-Newton iterations. When the first subproblem was solved to 
lyak and $A$. Sofer

slems. $\mu_{0}=10^{-3}$

$=1, i=1, \ldots, m$;

, terminated when

$\mathrm{d}$ was terminated

$-\dot{n}:$

is used to solve all ar problems.

e costs of running $\mathrm{g}$ the initial point ained problem are ie total number of a the line search), on to compute the

I performs notably e modified barrier and only $68 \%$ as $i$ is seen that the id on 9 of the 33 $=100,1024), 105$ e a by-product of blems. By seeking et. the behavior of by fine-tuning the performance from other problems). i we will only list the method that

id less accurately, ninated when the $=10^{-3}$ ) then the nated after a fixed lem was solved to

\begin{tabular}{|c|c|c|c|c|c|c|c|c|c|}
\hline \multirow[t]{2}{*}{ Problem } & \multirow[t]{2}{*}{$n$} & \multicolumn{4}{|c|}{ Modified } & \multicolumn{4}{|c|}{ Stabilized } \\
\hline & & it & Is & $\mathrm{cg}$ & total & it & is & $\mathrm{cg}$ & total \\
\hline 1 & 1000 & 78 & 258 & 432 & 690 & 49 & 83 & 212 & 295 \\
\hline 1 & 100 & 46 & 187 & 195 & 382 & 39 & 75 & 153 & 228 \\
\hline 2 & 1000 & 70 & 103 & 361 & 464 & 82 & 140 & 789 & 929 \\
\hline 2 & 100 & 33 & 46 & 86 & 132 & 44 & 68 & 144 & 212 \\
\hline 3 & 1000 & $i i$ & 144 & 448 & 592 & 97 & 167 & 834 & 1001 \\
\hline 3 & 100 & 50 & 103 & 201 & 304 & 59 & 88 & 279 & 367 \\
\hline 6 & 1000 & 48 & 88 & 166 & 254 & 114 & 192 & 1321 & 1513 \\
\hline 6 & 100 & 32 & 56 & 88 & 144 & 66 & 117 & 305 & 422 \\
\hline 8 & 1000 & 16 & 36 & 42 & 78 & 23 & 49 & 49 & 98 \\
\hline 9 & 100 & 42 & 86 & 165 & 251 & 114 & 284 & 301 & 585 \\
\hline 10 & 1000 & 45 & 68 & 174 & 242 & 87 & 212 & 316 & 528 \\
\hline 12 & 1000 & 69 & 146 & 434 & 580 & 57 & 116 & 448 & 564 \\
\hline 28 & 1000 & 19 & 25 & 57 & 82 & 16 & 36 & 46 & 82 \\
\hline 30 & 100 & 32 & 52 & 90 & 142 & 36 & 57 & 86 & 143 \\
\hline 31 & 100 & 38 & 128 & 112 & 240 & 53 & 119 & 152 & 271 \\
\hline 38 & 1000 & 60 & 128 & 324 & 452 & 76 & 135 & 501 & 636 \\
\hline 39 & 961 & 56 & 128 & 572 & 700 & 98 & 243 & 1134 & 1377 \\
\hline 40 & 1000 & 32 & 88 & 141 & 229 & 35 & 65 & 175 & 240 \\
\hline 41 & 1000 & 39 & 82 & 164 & 246 & 50 & 92 & 240 & 332 \\
\hline 42 & 1000 & 42 & 101 & 179 & 280 & 37 & 83 & 95 & 178 \\
\hline 43 & 1000 & 38 & 57 & 147 & 204 & 55 & 111 & 292 & 403 \\
\hline 45 & 1000 & +2 & 65 & 142 & 207 & 55 & 128 & 153 & 281 \\
\hline 48 & 1000 & 29 & 86 & 89 & 175 & 60 & 157 & 149 & 306 \\
\hline 49 & 1000 & 19 & 25 & 57 & 82 & 16 & 36 & 46 & 80 \\
\hline 50 & 1000 & 63 & 139 & 339 & 478 & 72 & 129 & 599 & 728 \\
\hline 51 & 1000 & 57 & 122 & 190 & 312 & 49 & 79 & 283 & 362 \\
\hline 52 & 1000 & 54 & 148 & 283 & 431 & 69 & 113 & 579 & 692 \\
\hline 54 & 1000 & 56 & 138 & 248 & 386 & 68 & 108 & 247 & 355 \\
\hline 55 & 1000 & 59 & 120 & 232 & 352 & 255 & 413 & 801 & 1214 \\
\hline 102 & 1024 & 91 & 151 & 685 & 836 & 82 & 134 & 573 & 707 \\
\hline 102 & 100 & 47 & 82 & 176 & 258 & 40 & 74 & 148 & 222 \\
\hline 105 & 1024 & 69 & 106 & 437 & 543 & 64 & 115 & 508 & 623 \\
\hline 105 & 100 & 44 & 69 & 157 & 226 & 37 & 71 & 120 & 191 \\
\hline Totals & & 1592 & 3361 & 7613 & 10974 & 2154 & 4089 & 12078 & 16165 \\
\hline
\end{tabular}

Table 2: Results using (a) modified barrier method, (b) stabilized barrier method plus enhancements. ${ }^{a}$

\footnotetext{
"Column "it" records the number of outer iterations, "ls" records the number of gradient evaluations used in the line search, "cg" records the number of gradient evaluations used in the inner iteration, and "total" records the total number of gradient evaluations ("ls" plus "cg")
} 
"full" accuracy $\left(\epsilon_{3}=10^{-6}\right)$, then the results were worse:

S. G. Nash, R. Polyak, and A. Sofe

\section{$\left(\begin{array}{llll}1608 & 3480 & 8519 & 11999\end{array}\right)$}

The overall convergence of the modified barrier method seems to be driven by the to get better initial Lagrange multiplier estimats subproblem less accurately we hope first subproblem is solved too crudely, however, it it relatively low expense. If the of the Lagrange multipliers. Solving the first subprobsible to obtain poor estimates wasteful, though, because it does not make senseblem to full accuracy can also be

with arbitrary Lagrange multipliers $\left(\lambda_{i}=1\right)$.
We experimented with "more to accurately solve a subproblem

plier estimates, trying to use gradient and choices of the initial Lagrange multito compute first-order multiplier estimates. The results were poor the initial point $x_{0}$
near 20,000 ). $\mu_{0}$ from the value used above $\left(\mu_{0}=10^{-3}\right)$, but choice of the initial barrier parameter unchanged. The following totals were obtained with $\mu_{0}=10^{-1}$ :

\section{$\left(\begin{array}{llll}2367 & 3928 & 10005 & 13933\end{array}\right)$}

with $\mu_{0}=10^{-2}$ :

$$
\text { with } \mu_{0}=10^{-4} \text { : }
$$

\section{$\left(\begin{array}{ll}1875 & 3364\end{array}\right.$}

8650 12014)

We also attempted to define $\left.\begin{array}{llll}2002 & 6836 & 12151 & 18987\end{array}\right)$

was done for the barrier function. This atty based on gradient information at $x_{0}$, as

Tests were also performed where the subprobled, with grand totals near 20,000 . ing $\epsilon_{3}>10^{-6}$ ). These were not successful thoblems were solved less accurately (usrequire accurate Lagrange multiplier estimates, The modified barrier method seems to solving the subproblems accurately.

Finally we experimen

surprisingly successful strategy on a darge nutes of reducing the barrier parameter. A the barrier parameter fixed at $\mu=10^{-3}$ for number of the test problems was to leave behaved poorly on a few subproblems, making subproblems. However, this strategy the barrier method more rapidly did not work well, in petitive overall. Reducing with the stabilized barrier method. We think thatl, in contrast to our experience the barrier parameter more quickly if some form that it might be possible to reduce found for the modified barrier method.

The strategies for running the two

bilized barrier method a larger number of suds effectively are different. In the stacoarsely, and the barrier parameter is reduced subproblems are used, each one solved other enhancements are then used to safeguard anly. Extrapolation techniques and 
se driven by the zurately we hope expense. If the n poor estimates iracy can also be ve a subproblem

Lagrange multi$e$ initial point $x_{0}$ vith grand totals arrier parameter rameter settings

mation at $x_{0}$, as tals near 20,000 . s accurately (usnethod seems to btained without

s parameter. A ms was to leave rr, this strategy erall. Reducing our experience ssible to reduce cedure could be

ent. In the staeach one solved 1 techniques and nethod. For the

\begin{tabular}{|c|c|c|c|c|c|c|c|c|c|}
\hline \multirow[t]{2}{*}{$\mu$} & \multicolumn{3}{|c|}{ Individual } & \multicolumn{4}{|c|}{ Cumulative } & \multirow[t]{2}{*}{ Gap } & \multirow[t]{2}{*}{$\nabla \mathcal{L}$} \\
\hline & it & Is & $\mathrm{cg}$ & it & Is & $\mathrm{cg}$ & total & & \\
\hline $1.46 \times 10^{5}$ & 1 & 2 & 2 & 1 & 2 & 2 & $\overline{4}$ & $6.8 \times 10^{-3}$ & $5.5 \times 10^{-6}$ \\
\hline $1.46 \times 10^{4}$ & 5 & 9 & 29 & 6 & 11 & 31 & 42 & $1.1 \times 10^{-2}$ & $6.7 \times 10^{-3}$ \\
\hline $1.46 \times 10^{3}$ & 6 & $i$ & 69 & 12 & 18 & 100 & 118 & $5.4 \times 10^{-3}$ & $1.4 \times 10^{-3}$ \\
\hline $1.46 \times 10^{2}$ & 5 & 7 & 30 & 17 & 25 & 130 & 155 & $2.6 \times 10^{-3}$ & $2.9 \times 10^{-4}$ \\
\hline $1.46 \times 10^{1}$ & 9 & 16 & 46 & 26 & 41 & 176 & 217 & $6.4 \times 10^{-4}$ & $3.5 \times 10^{-6}$ \\
\hline $1.46 \times 10^{0}$ & 11 & 18 & 62 & 37 & 59 & 238 & 297 & $8.5 \times 10^{-5}$ & $1.2 \times 10^{-5}$ \\
\hline $1.46 \times 10^{-1}$ & 7 & 12 & 28 & 44 & 71 & 266 & 337 & $8.8 \times 10^{-6}$ & $1.1 \times 10^{-5}$ \\
\hline $1.46 \times 10^{-2}$ & 3 & 4 & 13 & 47 & 75 & 279 & 354 & $8.8 \times 10^{-7}$ & $1.0 \times 10^{-5}$ \\
\hline $1.46 \times 10^{-3} *$ & 1 & 2 & 2 & 48 & 77 & 281 & 358 & $8.8 \times 10^{-8}$ & $2.8 \times 10^{-9}$ \\
\hline $1.46 \times 10^{-4} *$ & 1 & 2 & 2 & 49 & 79 & 283 & 362 & $8.8 \times 10^{-9}$ & $1.8 \times 10^{-10}$ \\
\hline
\end{tabular}

Table 3: Using the stabilized barrier method to solve problem 51 with $n=1000 .^{a}$

\footnotetext{
${ }^{a}$ An $*$ indicates subproblems where the 1 -inverse formula for the search direction was used. Column "it" records the number of outer iterations, "ls" records the number of gradient evaluations used in the line search. " $\mathrm{cg}$ " records the number of gradient evaluations used in the inner iteration, and "total" records the total number of gradient evaluations ("ls" plus "cg"). The column "Gap" records the (scaled) duality gap, and the column " $\|\nabla \mathcal{L}\|$ " records the norm of the (scaled) Lagrangian function.
}

modified barrier method, fewer subproblems are used, each one is solved accurately, the barrier parameter is reduced slowly (and frequently need not be reduced at all). In Tables 3 and 4 these points are illustrated, with the two methods being applied to problem 51 with $n=1000$.

For completeness we also illustrate the performance of a "naive" barrier method on the same problem. The "naive" barrier method is simply a barrier method without the special enhancements. It uses a line search based on inverse cubic interpolation, it does not use extrapolation, special initialization of $\mu$, or the 1 -inverse formula. and it does not save the preconditioners from one subproblem to the next. The results for this method are shown in Table 5 .

\section{Conclusions}

We have compared the performance of a stabilized barrier method with the performance of a modified barrier method. Our past experience indicates that the stabilized barrier method is a robust and effective method for solving bound-constrained prob- 


\begin{tabular}{|c|rrr|rrrr|c|c|c|}
\hline$\mu$ & \multicolumn{7}{|c|}{ Individual } & \multicolumn{7}{|c|}{ Cumulative } & Gap & $\nabla \mathcal{L}$ & Infeas \\
& it & ls & $\mathrm{cg}$ & it & ls & $\mathrm{cg}$ & tot & & & \\
\hline $1.00 \times 10^{-3}$ & 13 & 23 & 55 & 13 & 23 & 55 & 78 & $1.1 \times 10^{-2}$ & $2.1 \times 10^{-3}$ & $5.7 \times 10^{-2}$ \\
$5.00 \times 10^{-4}$ & 17 & 25 & 56 & 30 & 48 & 111 & 159 & $1.6 \times 10^{-4}$ & $5.5 \times 10^{-7}$ & $1.2 \times 10^{-3}$ \\
$2.50 \times 10^{-4}$ & 15 & 45 & 45 & 45 & 93 & 156 & 249 & $1.1 \times 10^{-6}$ & $1.5 \times 10^{-6}$ & $7.9 \times 10^{-6}$ \\
$1.25 \times 10^{-4}$ & 7 & 8 & 20 & 52 & 101 & 176 & 277 & $3.7 \times 10^{-9}$ & $3.5 \times 10^{-7}$ & $2.7 \times 10^{-8}$ \\
$6.25 \times 10^{-5}$ & 5 & 21 & 14 & 57 & 122 & 190 & 312 & $7.4 \times 10^{-11}$ & $7.7 \times 10^{-7}$ & $7.6 \times 10^{-10}$ \\
\hline
\end{tabular}
Table 4: Using the modified barrier method to solve problem 51 with
\[ n=1000^{\circ} \]

\footnotetext{
"Column "it" records the number of outer iterations. "Is" records the number of gradient evaluations used in the line search, "cg" records the the total gradient evaluations used in the inner iteration, and "tot" records "Gap" records of gradient evaluations ("ls" plus "cg"). The column norm of the (scaled) Lagrangian fun gap, the column " $\|\nabla \mathcal{L}\|$ " records the the infinity norm of the infoasibities wion, and the column "Infeas" records the infeasibilities with respect to the bound constraints.
}

\begin{tabular}{|l|rrr|rrrr|c|c|}
\hline$\mu$ & \multicolumn{6}{|c|}{ Individual } & \multicolumn{5}{|c|}{ Cumulative } & Gap & $\nabla \mathcal{L}$ \\
& it & ls & $\mathrm{cg}$ & it & $\mathrm{ls}$ & $\mathrm{cg}$ & total & & \\
\hline $1.00 \times 10^{2}$ & 15 & 55 & 59 & 15 & 55 & 59 & 114 & $2.0 \times 10^{-3}$ & $3.8 \times 10^{-6}$ \\
$1.00 \times 10^{1}$ & 19 & 106 & 65 & 34 & 161 & 124 & 285 & $4.7 \times 10^{-4}$ & $3.6 \times 10^{-7}$ \\
$1.00 \times 10^{0}$ & 22 & 72 & 98 & 56 & 233 & 222 & 455 & $5.8 \times 10^{-5}$ & $8.7 \times 10^{-9}$ \\
$1.00 \times 10^{-1}$ & 20 & 122 & 107 & 76 & 355 & 329 & 684 & $6.0 \times 10^{-6}$ & $5.1 \times 10^{-8}$ \\
$1.00 \times 10^{-2}$ & 19 & 69 & 104 & 95 & 424 & 433 & 857 & $6.0 \times 10^{-7}$ & $4.3 \times 10^{-8}$ \\
$1.00 \times 10^{-3}$ & 19 & 102 & 118 & 114 & 526 & 551 & 1077 & $6.0 \times 10^{-8}$ & $8.1 \times 10^{-8}$ \\
$1.00 \times 10^{-4}$ & 19 & 59 & 97 & 133 & 585 & 648 & 1233 & $6.0 \times 10^{-9}$ & $2.7 \times 10^{-8}$ \\
\hline
\end{tabular}

Table 5: Using a naive barrier method to solve problem 51 with $n=1000 .^{a}$

\footnotetext{
"Column "it" records the number of outer iterations, "ls" records the number of gradient evaluations used in the line search, "cg" records the number of gradient evaluations used in the inner iteration, and "total" records the total number of gradient evaluaality gap, and "cg"). The column "Gap" records the (scaled) duLagrangian function.
} 


\begin{tabular}{|c|c|}
\hline & Infeas \\
\hline$j^{-3}$ & $5.7 \times 10^{-2}$ \\
$j^{-7}$ & $1.2 \times 10^{-3}$ \\
$j^{-6}$ & $7.9 \times 10^{-6}$ \\
$j^{-i}$ & $2.7 \times 10^{-8}$ \\
$j^{-7}$ & $7.6 \times 10^{-10}$ \\
\hline
\end{tabular}

in 51 with

records the records the "tot" records The column records the feas" records 1 constraints.

\begin{tabular}{|c|c|}
\hline & $\nabla \mathcal{L}$ \\
\hline 3 & $3.8 \times 10^{-6}$ \\
+ & $3.6 \times 10^{-i}$ \\
5 & $8.7 \times 10^{-9}$ \\
6 & $5.1 \times 10^{-8}$ \\
$i$ & $4.3 \times 10^{-8}$ \\
3 & $8.1 \times 10^{-8}$ \\
& $2.7 \times 10^{-8}$ \\
\hline
\end{tabular}

jI with

ns. "Is"

e search.

the inner

it evalua-

aled) du-

e (scaled) lems. Our software for the stabilized barrier method is a result of much testing and enhancement, and represents a considerable improvement over "naive" barrier techniques. In contrast, our software for the modified barrier method is less sophisticated. Nevertheless, its performance is superior to the stabilized barrier method on the bound-constrained problems that we have tested. We expect that we may obtain even better performance with further testing and enhancement. This suggests that modified barrier methods are a promising tool for solving large nonlinear programming problems.

\section{References}

(1) B. M. Averick, R. G. Carter, and J. J. Moré, (1991), "The Minpack-2 Test Problem Collection (Preliminary Version)," Report ANL/MCS-TM-150, Mathematics and Computer Science Division, Argonne National Laboratory, Argonne IL.

[2] A. Ben-Tal, I. Yuzefovich and M. Zibulevsky, (1992), "Penalty/Barrier Muitiplier Methods for minimax and constrained smooth convex problems," Research Report 9/92. Optimization Laboratory, Faculty of Industrial Engineering and Management, Technion (Israel Institute of Technology).

(3) M. G. Breitfeld and D. F. Shanno, (1993), "Preliminary computational experience with modified log-barrier functions for large-scale nonlinear programming," in Large Scale Optimization: State of the Art, (W. W. Hager, D. W. Hearn and P.... Pardalos, Eds.), Kluwer Academic Publishers B. V..

4] A. R. Conn. N. I. M. Gould, and P. L. Toint, (1988), "Testing a class of methods for solving minimization problems with simple bounds on the variables," Math. Comp., 50, 399- $\$ 30$.

[5] A. V. Fiacco and G. P. McCormick, (1968), Nonlinear Programming: Sequential Unconstrained Minimization Techniques, John Wiley and Sons, New York (This book has been reprinted by SIAM, Philadelphia, 1990).

(6) P. E. Gill. W. Murray, and M. H. Wright, (1981), Practical Optimization, Academic Press. New York.

[7] D. L. Jensen and R. Polyak, (1992), "The convergence of a modified barrier method for convex programming," Technical Report 18570, IBM Thomas J. Watson Research Center, Yorktown Heights, NY.

[8] W. Murray, (1971), "Analytical expressions for the eigenvalues and eigenvectors of the Hessian matrices of barrier and penalty functions," J. Opt. Th. App.. 7, $189-196$. 
[9] W. Murray and M. H. Wright, (1976), "Efficient linear search algorithms for the logarithmic barrier function," Report SOL 76-18, Dept. of Operations Research, Stanford University, Stanford CA.

[10] S. G. Nash, (1993), "A parallel barrier algorithm for large-scale bound-constrained optimization," Technical Report 93-01, Operations Research and Engineering Department, George Mason University, Fairfax VA.

[11] S. G. Nash and J. Nocedal, (1991), "A Numerical Study of the Limited Memory BFGS Method and the Truncated-Newton Method for Large Scale Optimization," SIAM J. Opt., 1, 358-372.

[12] S. G. Nash and A. Sofer, (1990), "Assessing a search direction within a truncatedNewton method," Operations Research Letters, 9, 219-221.

[13] S. G. Nash and A. Sofer, (1991), "A general-purpose parallel algorithm for unconstrained optimization," SIAM J. Opt, 1, 530-547.

[14] S. G. Nash and A. Sofer, (1993), "A barrier method for large-scale constrained optimization," ORSA Journal on Computing, 5, 40-53.

[15] S. G. Nash and A. Sofer, (1993), "Preconditioning of reduced matrices," Technical Report, Operations Research and Engineering Department, George Mason
University, Fairfax VA.

[16] J. M. Ortega and W. C. Rheinboldt, (1970), Iterative Solution of Nonlinear Equations in Several Variables, Academic Press, London and New York.

[1i] R. Polyak, (1992), "Modified barrier functions (theory and methods)," Mathe. matical Programming, 54, 177-222.

[1S] M. H. Wright, (1992), "Determining subspace information from the Hessian of the barrier function," Numerical Analysis Manuscript 92-02, AT\& T Bell Laboratories, Murray Hill NJ. 